\title{
On the deformed oscillator and the deformed derivative associated with the Tsallis $q$-exponential
}

\author{
Ramaswamy Jagannathan* \\ * The Institute of Mathematical Sciences \\ 4 th Cross Street, Central Institutes of Technology (CIT) Campus \\ Tharamani, Chennai 600113, India \\ $\$$ Department of Mathematics and Sciences \\ College of Arts and Applied Sciences (CAAS), Dhofar University \\ Post Box No. 2509, Postal Code: 211, Salalah, Oman
}

\begin{abstract}
The Tsallis $q$-exponential function $e_{q}(x)=(1+(1-q) x)^{\frac{1}{1-q}}$ is found to be associated with the deformed oscillator defined by the relations $\left[N, a^{\dagger}\right]=a^{\dagger},[N, a]=-a$, and $\left[a, a^{\dagger}\right]=\phi_{T}(N+1)-\phi_{T}(N)$, with $\phi_{T}(N)=N /(1+(q-1)(N-1))$. In a Bargmann-like representation of this deformed oscillator the annihilation operator $a$ corresponds to a deformed derivative with the Tsallis $q$-exponential functions as its eigenfunctions, and the Tsallis $q$-exponential functions become the coherent states of the deformed oscillator. When $q=2$ these deformed oscillator coherent states correspond to states known variously as phase coherent states, harmonious states, or pseudothermal states. Further, when $q=1$ this deformed oscillator is a canonical boson oscillator, when $1<q<2$ its ground state energy is same as for a boson and the excited energy levels lie in a band of finite width, and when $q \longrightarrow 2$ it becomes a two-level system with a nondegenerate ground state and an infinitely degenerate excited state.
\end{abstract}

Keywords: Nonextensive statistical mechanics, Tsallis $q$-exponential, deformed exponentials, deformed oscillators, deformed numbers, deformed derivatives, nonlinear coherent states, $f$-oscillators, $f$-coherent states, phase coherent states.

\footnotetext{
${ }^{\S}$ Retired Faculty (Physics); email: jagan@imsc.res.in ORCID ID: https://orcid.org/0000-0003-2968-2044

ฯemail: rohelakhan@yahoo.com ORCID ID: https://orcid.org/0000-0003-1264-2302
} 


\section{Introduction}

A posteriori knowledge gained from special relativity and quantum mechanics suggests that it may be possible to build models in physics actually realizable in Nature by mathematically deforming existing theories (see, e.g., [1] and references therein). We may look at the attempts to build $q$-deformed models in physics using $q$-deformations of the mathematical structures associated with the existing models from this point of view. There have been two totally different $q$-deformation schemes in physics running in parallel since 1980s: One emerged, essentially, from studies on quantum algebras (see, e.g., [2] and references therein) and the other stemmed from the pioneering paper of Tsallis [3] on nonextensive statistical mechanics.

Studies on the deformation of quantum commutation relations started in 1970s from different points of view (see [4] [ see [7] for an account of the early history of deformed quantum commutation relations). With the advent of quantum algebras in 1980s, representation theory of quantum algebras led to the $q$-deformed quantum harmonic oscillator, or the so called $q$-oscillator [8 11]. Harmonic oscillator being a basic paradigm playing a central role in modeling various physical phenomena $q$-oscillators and their generalizations have been studied extensively. Deformed oscillators and related mathematical structures have been used in building models in several areas of physics: nuclear structure physics using models like shell model, interacting boson model, etc., vibrational-rotational molecular spectroscopy, statistics of deformed oscillators, statistics interpolating between the Bose statistics and the Fermi statistics, deformed thermodynamics and applications to condensed matter physics, nonclassical states in quantum optics, noncommutative probability theory, information theory, etc. (see, e.g., [12 27], and references therein). In the discussion of vibrational-rotational spectroscopy, besides the $q$-deformation of the quantum harmonic oscillator, the quantum rigid rotator is $q$-deformed based on the $q$-deformation of the Lie algebra of angular momentum operators.

The other $q$-deformation scheme follows from the nonextensive entropy

$$
S_{q}=k\left(\frac{1-\sum_{i=1}^{W} p_{i}^{q}}{q-1}\right), \quad \text { with } \sum_{i=1}^{W} p_{i}=1
$$

introduced by Tsallis in [3] where $W$ is the total number of possible configurations of the system under consideration, $p_{i}$ is the probability that the 
system is in the $i$ th configuration, and $q$ is a real parameter. Defining

$$
\ln _{q} x=\frac{x^{1-q}-1}{1-q}
$$

the Tsallis $q$-logarithm, we get

$$
S_{q}=k \sum_{i=1}^{W} p_{i} \ln _{q}\left(p_{i}^{-1}\right)
$$

such that

$$
\lim _{q \longrightarrow 1} S_{q}=-k \sum_{i=1}^{W} p_{i} \ln p_{i}
$$

the Boltzmann-Gibbs-Shannon entropy. From (2) we have

$$
x=\left(1+(1-q) \ln _{q} x\right)^{\frac{1}{1-q}} .
$$

Thus, if we define

$$
e_{q}(x)=(1+(1-q) x)^{\frac{1}{1-q}}
$$

the Tsallis $q$-exponential function, then

$$
e_{q}\left(\ln _{q} x\right)=x
$$

Note that

$$
\lim _{q \longrightarrow 1} \ln _{q} x=\ln x, \quad \lim _{q \longrightarrow 1} e_{q}(x)=e^{x} .
$$

It is to be observed that in the definition of the ordinary exponential function,

$$
e^{x}=\lim _{N \longrightarrow \infty}\left(1+\frac{x}{N}\right)^{N}
$$

if we replace the inifinitesimally small $1 / N$ corresponding to large $N$ by $1-q$, with $0<1-q=\epsilon \approx 0$, then we get the Tsallis $q$-exponential function (6)). Having understood $e_{q}(x)$ in this way its definition can be extended to other values of $q,<1$ or $>1$. In general, for $q \neq 1$ the definition is

$$
e_{q}(x)=\left\{\begin{array}{l}
(1+(1-q) x)^{\frac{1}{1-q}} \text { if } 1+(1-q) x \geq 0 \\
0 \text { otherwise }
\end{array}\right.
$$


It follows that $e_{q}(0)=1,0 \leq e_{q}(x) \leq \infty$, and

$$
\frac{d e_{q}(x)}{d x}=\left(e_{q}(x)\right)^{q} .
$$

It is not surprising that being a natural deformation of $e^{x}$ the Tsallis $q$ exponential function $e_{q}(x)$ and its inverse function $\ln _{q}(x)$ find applications in several areas of physics and other sciences like nonequilibrium processes, anomalous diffusion, turbulence, spin-glasses, nonlinear dynamics, high energy particle collisions, dissipative optical lattices, atmospheric physics, astrophysics, biological systems, medical imaging, earthquake studies, neural networks, economics, stock markets, etc. (see, e.g., 28 32 and references therein, and the extensive bibliography in [33]).

It should be noted that in nonextensive statistical mechanics and its applications one defines

$$
e_{q}(k x)=(1+(1-q) k x)^{\frac{1}{1-q}}
$$

and $e_{q}(k x) \neq e_{q}(x)^{k}$. For example, to define the $q$-Gaussian function one takes $e_{q}\left(-\beta x^{2}\right)=\left(1-(1-q) \beta x^{2}\right)^{1 /(1-q)}$. Carlitz introduced a deformed analogue of the exponential while defining the socalled degenerate Bernoulli numbers and polynomials (see [34,35]). The deformed exponential function of Carlitz is defined by $\exp _{\lambda}(x)=(1+\lambda x)^{1 / \lambda}$ and $\exp _{\lambda}(t x)=\left(\exp _{\lambda}(x)\right)^{t}=$ $(1+\lambda x)^{t / \lambda}$. This difference between the deformed exponential of Carlitz and the Tsallis $q$-exponential is crucial in applications (see, e.g., [36]).

In this paper we are concerned only with the mathematical structures of $q$-deformations of the quantum harmonic oscillator. The canonical harmonic oscillator, or the boson, is defined by the commutation relations

$$
\left[N, b^{\dagger}\right]=b^{\dagger}, \quad[N, b]=-b, \quad\left[b, b^{\dagger}\right]=1,
$$

where $b^{\dagger}, b$ and $N$ are the boson creation, annihilation and number operators, respectively. These relations imply that $b^{\dagger} b=N$ and $b b^{\dagger}=N+1$. The Fock representation of the boson algebra (13) is given by

$b^{\dagger}|n\rangle=\sqrt{n+1}|n+1\rangle, \quad b|n\rangle=\sqrt{n}|n-1\rangle, \quad N|n\rangle=n|n\rangle, \quad n=0,1,2, \ldots$,

with $\{|n\rangle \mid n=0,1,2, \ldots\}$ being an orthonormal basis. Starting with the ground state $|0\rangle$ we can build the higher states as

$$
|n\rangle=\frac{b^{\dagger^{n}}}{\sqrt{n !}}|0\rangle .
$$


The Hamiltonian of the boson oscillator is

$$
H=\left(b^{\dagger} b+\frac{1}{2}\right)=\frac{1}{2}\left(b b^{\dagger}+b^{\dagger} b\right),
$$

with the energy spectrum

$$
E_{n}=n+\frac{1}{2}, \quad n=0,1,2, \ldots,
$$

such that

$$
H|n\rangle=E_{n}|n\rangle .
$$

Coherent states are eigenfunctions of $b$ :

$$
b|\alpha\rangle=\alpha|\alpha\rangle,
$$

where $\alpha$ is any complex number. The solution for $|\alpha\rangle$ is

$$
|\alpha\rangle=\frac{1}{\sqrt{e^{|\alpha|^{2}}}} \sum_{n=0}^{\infty} \frac{\alpha^{n}}{\sqrt{n !}}|n\rangle,
$$

with the normalization $\langle\alpha \mid \alpha\rangle=1$. We can write

$$
|\alpha\rangle=\frac{1}{\sqrt{e^{|\alpha|^{2}}}} e^{\alpha b^{\dagger}}|0\rangle
$$

In the Bargmann-like representation

$$
b^{\dagger}=x, \quad b=D=\frac{d}{d x}, \quad N=x D,
$$

and the monomials

$$
\xi_{n}(x)=\frac{x^{n}}{\sqrt{n !}}, \quad n=0,1,2, \ldots,
$$

carry the Fock representation (14) as given by

$$
b^{\dagger} \xi_{n}=\sqrt{n+1} \xi_{n+1}, \quad b \xi_{n}=\sqrt{n} \xi_{n-1}, \quad N \xi_{n}=n \xi_{n}, \quad n=0,1,2, \ldots .
$$

The monomials $\left\{\xi_{n}(x) \mid n=0,1,2, \ldots\right\}$ are seen to form an orthonormal basis with respect to the inner product

$$
\langle f \mid g\rangle=\left.\left[f^{*}(D) g(x)\right]\right|_{x=0} .
$$


Note that in this representation $e^{\alpha x}$ is an eigenfunction of $b$ for any complex number $\alpha$. Under the inner product (25) it is seen that $\left\langle e^{\alpha x} \mid e^{\alpha x}\right\rangle=e^{|\alpha|^{2}}$. Thus, in this representation, the coherent states are given by

$$
\psi_{\alpha}(x)=\frac{1}{\sqrt{e^{|\alpha|^{2}}}} e^{\alpha x}=\frac{1}{\sqrt{e^{|\alpha|^{2}}}} \sum_{n=0}^{\infty} \frac{\alpha^{n}}{\sqrt{n !}} \xi_{n}(x),
$$

which are normalized as $\left\langle\psi_{\alpha} \mid \psi_{\alpha}\right\rangle=1$.

In general, a deformed oscillator algebra is prescribed by the relations

$$
\begin{aligned}
{\left[N, a^{\dagger}\right] } & =a^{\dagger}, \quad[N, a]=-a, \\
{\left[a, a^{\dagger}\right] } & =\phi(N+1)-\phi(N), \quad \text { or } \quad a a^{\dagger}=\phi(N+1), \quad a^{\dagger} a=\phi(N),
\end{aligned}
$$

where $a^{\dagger}, a$, and $N$ are the deformed oscillator creation, annihilation, and number operators, respectively. The real function $\phi(n)$, sometimes called the deformation, or structure, function, characterizes the deformed oscillator. Note that the first two relations of (27) imply that $a^{\dagger} a$ and $a a^{\dagger}$ commute with $N$. In the case of the canonical boson oscillator (13) $\phi(N)=N$.

The Fock representation of the algebra (27) can be constructed easily as follows:

$$
\begin{aligned}
a^{\dagger}|n\rangle & =\sqrt{\phi(n+1)}|n+1\rangle, \quad a|n\rangle=\sqrt{\phi(n)}|n-1\rangle, \\
N|n\rangle & =n|n\rangle, \quad n=0,1,2, \ldots,
\end{aligned}
$$

Note that $a^{\dagger} a|n\rangle=\phi(n)|n\rangle$. Since $a|0\rangle=0$, we must have $\phi(0)=0$. Now, defining

$$
\phi(0) !=1, \quad \phi(n) !=\prod_{j=1}^{n} \phi(j), \quad n=1,2, \ldots
$$

we can write

$$
|n\rangle=\frac{a^{\dagger^{n}}}{\sqrt{\phi(n) !}}|0\rangle .
$$

The Hamiltonian of the deformed oscillator is taken to be

$$
H_{\phi}=\frac{1}{2}\left(a a^{\dagger}+a^{\dagger} a\right)
$$

generalizing (16). The energy spectrum of the deformed oscillator becomes

$$
E_{n, \phi}=\frac{1}{2}(\phi(n+1)+\phi(n))
$$


generalizing (17), and

$$
H_{\phi}|n\rangle=E_{n, \phi}|n\rangle .
$$

Associated to any $\phi(n)$ let us define the deformed exponential function

$$
e_{\phi}^{x}=\sum_{n=0}^{\infty} \frac{x^{n}}{\phi(n) !}
$$

which we shall refer to as the $\phi$-exponential function. It can be verified that the normalized coherent states of the deformed oscillator (27), eigenstates of $a$, are given by

$$
|\alpha\rangle_{\phi}=\frac{1}{\sqrt{e_{\phi}^{|\alpha|^{2}}}} \sum_{n=0}^{\infty} \frac{\alpha^{n}}{\sqrt{\phi(n) !}}|n\rangle=\frac{1}{\sqrt{e_{\phi}^{|\alpha|^{2}}}} e_{\phi}^{\alpha a^{\dagger}}|0\rangle,
$$

in which the complex number $\alpha$ is such that $e_{\phi}^{|\alpha|^{2}}<\infty$. Let

$$
D_{\phi} F(x)=\left[\frac{1}{x} \phi(x D)\right] F(x) .
$$

Note that as a generalization of the relation

$$
D x^{n}=n x^{n-1} .
$$

we have

$$
D_{\phi} x^{n}=\phi(n) x^{n-1} .
$$

The Bargmann-like representation is

$$
a^{\dagger}=x, \quad a=D_{\phi}, \quad N=x D .
$$

so that the monomials

$$
\xi_{n, \phi}(x)=\frac{x^{n}}{\sqrt{\phi(n) !}}, \quad n=0,1,2, \ldots,
$$

carry the Fock representation (28) as given by

$$
\begin{aligned}
a^{\dagger} \xi_{n, \phi} & =\sqrt{\phi(n+1)} \xi_{n+1, \phi}, \quad a \xi_{n, \phi}=\sqrt{\phi(n)} \xi_{n-1, \phi}, \\
N \xi_{n, \phi} & =n \xi_{n, \phi}, \quad n=0,1,2, \ldots .
\end{aligned}
$$


The monomials $\left\{\xi_{n, \phi}(x) \mid n=0,1,2, \ldots\right\}$ form an orthonormal basis with respect to the inner product

$$
\langle f \mid g\rangle_{\phi}=\left.\left[f^{*}\left(D_{\phi}\right) g(x)\right]\right|_{x=0} .
$$

Note that in this representation $e_{\phi}^{\alpha x}$ is an eigenfunction of $a$ for the complex number $\alpha$. Under the inner product (42)

$$
\left\langle e_{\phi}^{\alpha x} \mid e_{\phi}^{\alpha x}\right\rangle=e_{\phi}^{|\alpha|^{2}}
$$

Thus, in this representation, the coherent states are given by

$$
\psi_{\alpha, \phi}(x)=\frac{1}{\sqrt{e_{\phi}^{|\alpha|^{2}}}} e_{\phi}^{\alpha x}=\frac{1}{\sqrt{e_{\phi}^{|\alpha|^{2}}}} \sum_{n=0}^{\infty} \frac{\alpha^{n}}{\sqrt{\phi(n) !}} \xi_{n, \phi}(x),
$$

with the normalization $\left\langle\psi_{\alpha, \phi} \mid \psi_{\alpha, \phi}\right\rangle=1$, for any $\alpha$ such that $e_{\phi}^{|\alpha|^{2}}<\infty$. This leads to the result that the eigenfunctions of the deformed derivative $D_{\phi}$, or $a$, are the deformed exponential functions $e_{\phi}^{\alpha x}$ representing, apart from the normalization factors, the corresponding coherent states of the deformed oscillator in the Bargmann-like representation. The deformed derivative $D_{\phi}$ has been used to develop a very general theory of deformation of classical hypergeometric functions [37].

Now, an interesting question is whether there exists a deformed oscillator with which the Tsallis $q$-exponential function (6) is associated such that corresponding coherent states are given by $\left\{e_{q}\left(\alpha a^{\dagger}\right)|0\rangle \mid \alpha \in \mathbb{C}\right\}$ apart from the normalization factors. Or, in other words, is there a deformed oscillator for which in the Bargmann-like representation the annihilation operator $a$ will be a deformed derivative with the Tsallis $q$-exponential functions as its eigenfunctions. In the following we present the deformed oscillator and the deformed derivative associated with the Tsallis $q$-exponential function, after reviewing briefly some examples of deformed oscillators.

\section{Deformed oscillators: Some examples}

The $q$-oscillator with

$$
\begin{aligned}
{\left[N, a^{\dagger}\right] } & =a^{\dagger}, \quad[N, a]=-a, \quad\left[a, a^{\dagger}\right]=\phi(N+1)-\phi(N), \\
\phi(N) & =\frac{1-q^{N}}{1-q}=[N]_{q},
\end{aligned}
$$


was studied much before the advent of quantum groups (see [4-7]). Since

$$
[N+1]_{q}-q[N]_{q}=1
$$

the relation between $a$ and $a^{\dagger}$ is written as

$$
a a^{\dagger}-q a^{\dagger} a=1
$$

The Fock representation is

$$
\begin{aligned}
a^{\dagger}|n\rangle & =\sqrt{[n+1]_{q}}|n+1\rangle, \quad a|n\rangle=\sqrt{[n]_{q}}|n-1\rangle, \\
N|n\rangle & =n|n\rangle, \quad n=0,1,2, \ldots
\end{aligned}
$$

where

$$
[n]_{q}=\frac{1-q^{n}}{1-q}
$$

is Heine's basic number, or $q$-number (see, e.g., [38, 39]). Note that

$$
[0]_{q}=0, \quad[1]_{q}=1, \quad \lim _{q \longrightarrow 1}[n]_{q}=n .
$$

Now, with the definitions

$$
[0]_{q} !=1, \quad[n]_{q} !=\prod_{j=1}^{n}[j]_{q}, \quad n=1,2, \ldots
$$

we have

$$
|n\rangle=\frac{a^{\dagger^{n}}}{\sqrt{[n]_{q} !}}|0\rangle .
$$

Defining the original $q$-exponential function

$$
e_{q}^{x}=\sum_{n=0}^{\infty} \frac{x^{n}}{[n]_{q} !}
$$

the normalized coherent states of the $q$-oscillator (45) are given by

$$
|\alpha\rangle_{q}=\frac{1}{\sqrt{e_{q}^{|\alpha|^{2}}}} \sum_{n=0}^{\infty} \frac{\alpha^{n}}{\sqrt{[n]_{q} !}}|n\rangle=\frac{1}{\sqrt{e_{q}^{|\alpha|^{2}}}} e_{q}^{\alpha a^{\dagger}}|0\rangle .
$$


In the Bargmann-like representation

$$
a^{\dagger}=x, \quad a=D_{q}=\frac{1}{x}[x D]_{q}=\frac{1}{x}\left(\frac{1-q^{x D}}{1-q}\right), \quad N=x D,
$$

where $D_{q}$ is the Jackson derivative, or $q$-derivative, operator (see, e.g., [38, 39]) such that

$$
D_{q} F(x)=\frac{F(x)-F(q x)}{(1-q) x} .
$$

Note that

$$
D_{q} x^{n}=[n]_{q} x^{n-1} .
$$

In the Bargmann-like representation the monomials

$$
\xi_{n, q}(x)=\frac{x^{n}}{\sqrt{[n]_{q} !}}, \quad n=0,1,2, \ldots,
$$

carry the Fock representation of the $q$-oscillator algebra (45) as given by

$$
\begin{aligned}
& a^{\dagger} \xi_{n, q}=\sqrt{[n+1]_{q}} \xi_{n+1, q}, \quad a \xi_{n, q}=\sqrt{[n]_{q}} \xi_{n-1, q}, \\
& N \xi_{n, q}=n \xi_{n, q}, \quad n=0,1,2, \ldots
\end{aligned}
$$

The monomials $\left\{\xi_{n, q}(x) \mid n=0,1,2, \ldots\right\}$ form an orthonormal basis with respect to the inner product

$$
\langle f \mid g\rangle_{q}=\left.\left[f^{*}\left(D_{q}\right) g(x)\right]\right|_{x=0} .
$$

It follows from (57) that the $q$-exponential function

$$
e_{q}^{\alpha x}=\sum_{n=0}^{\infty} \frac{\alpha^{n} x^{n}}{[n]_{q} !}
$$

for any complex number $\alpha$, becomes an eigenfunction of $a$, and the normalized coherent states of the $q$-oscillator are given by

$$
\psi_{\alpha, q}(x)=\frac{1}{\sqrt{e_{q}^{|\alpha|^{2}}}} e_{q}^{\alpha x}=\frac{1}{\sqrt{e_{q}^{|\alpha|^{2}}}} \sum_{n=0}^{\infty} \frac{\alpha^{n}}{\sqrt{[n]_{q} !}} \xi_{n, q}(x) .
$$


The development of quantum groups and the representation theory of associated quantum algebras led first to the $\left(q^{-1}, q\right)$-oscillator [8, 9] (see also [10, 11]):

$$
\begin{aligned}
{\left[N, a^{\dagger}\right] } & =a^{\dagger}, \quad[N, a]=-a, \quad\left[a, a^{\dagger}\right]=\phi(N+1)-\phi(N), \\
\phi(N) & =\frac{q^{-N}-q^{N}}{q^{-1}-q}=[N]_{\left(q^{-1}, q\right)} .
\end{aligned}
$$

With the relation

$$
[N+1]_{\left(q^{-1}, q\right)}-q^{-1}[N]_{\left(q^{-1}, q\right)}=q^{N}
$$

the relation between $a$ and $a^{\dagger}$ becomes

$$
a a^{\dagger}-q^{-1} a^{\dagger} a=q^{N}
$$

Note the $q \longleftrightarrow q^{-1}$ symmetry. The Fock representation is

$$
\begin{aligned}
a^{\dagger}|n\rangle & =\sqrt{[n+1]_{\left(q^{-1}, q\right)}}|n+1\rangle, \quad a|n\rangle=\sqrt{[n]_{\left(q^{-1}, q\right)}}|n-1\rangle, \\
N|n\rangle & =n|n\rangle, \quad n=0,1,2, \ldots,
\end{aligned}
$$

where

$$
[n]_{\left(q^{-1}, q\right)}=\frac{q^{-n}-q^{n}}{q^{-1}-q}
$$

defines the $\left(q^{-1}, q\right)$-basic number. Note that

$$
[0]_{\left(q^{-1}, q\right)}=0, \quad[1]_{\left(q^{-1}, q\right)}=1, \quad \lim _{q \longrightarrow 1}[n]_{\left(q^{-1}, q\right)}=n .
$$

Now, with the definitions

$$
[0]_{\left(q^{-1}, q\right)} !=1, \quad[n]_{\left(q^{-1}, q\right)} !=\prod_{j=1}^{n}[j]_{\left(q^{-1}, q\right)}, \quad n=1,2, \ldots
$$

we have

$$
|n\rangle=\frac{a^{\dagger^{n}}}{\sqrt{[n]_{\left(q^{-1}, q\right)}}}|0\rangle
$$

Defining the $\left(q^{-1}, q\right)$-exponential function as

$$
e_{\left(q^{-1}, q\right)}^{x}=\sum_{n=0}^{\infty} \frac{x^{n}}{[n]_{\left(q^{-1}, q\right)} !}
$$


the normalized coherent states of the $\left(q^{-1}, q\right)$-oscillator (63) are given by

$$
|\alpha\rangle_{\left(q^{-1}, q\right)}=\frac{1}{\sqrt{e_{\left(q^{-1}, q\right)}^{|\alpha|^{2}}}} \sum_{n=0}^{\infty} \frac{\alpha^{n}}{\sqrt{[n]_{\left(q^{-1}, q\right)} !}}|n\rangle=\frac{1}{\sqrt{e_{\left(q^{-1}, q\right)}^{|\alpha|^{2}}}} e_{\left(q^{-1}, q\right)}^{\alpha a^{\dagger}}|0\rangle .
$$

The Bargmann-like representation is given by

$$
a^{\dagger}=x, \quad a=D_{\left(q^{-1}, q\right)}=\frac{1}{x}\left(\frac{q^{-x D}-q^{x D}}{q^{-1}-q}\right), \quad N=x D .
$$

Note that

$$
D_{\left(q^{-1}, q\right)} F(x)=\frac{F\left(q^{-1} x\right)-F(q x)}{\left(q^{-1}-q\right) x}, \quad D_{\left(q^{-1}, q\right)} x^{n}=[n]_{\left(q^{-1}, q\right)} x^{n-1} .
$$

Then, the monomials

$$
\xi_{n,\left(q^{-1}, q\right)}(x)=\frac{x^{n}}{\sqrt{[n]_{\left(q^{-1}, q\right) !}}}, \quad n=0,1,2, \ldots
$$

carry the Fock representation of the $\left(q^{-1}, q\right)$-oscillator algebra $(\underline{63})$ as given by

$$
\begin{aligned}
a^{\dagger} \xi_{n,\left(q^{-1}, q\right)} & =\sqrt{[n+1]_{\left(q^{-1}, q\right)}} \xi_{n+1,\left(q^{-1}, q\right)}, \\
a \xi_{n,\left(q^{-1}, q\right)} & =\sqrt{[n]_{\left(q^{-1}, q\right)}} \xi_{n-1,\left(q^{-1}, q\right)}, \\
N \xi_{n,\left(q^{-1}, q\right)} & =n \xi_{n,\left(q^{-1}, q\right)}, \quad n=0,1,2, \ldots .
\end{aligned}
$$

The monomials $\left\{\xi_{n,\left(q^{-1}, q\right)}(x) \mid n=0,1,2, \ldots\right\}$ form an orthonormal basis with respect to the inner product

$$
\langle f \mid g\rangle_{\left(q^{-1}, q\right)}=\left.\left[f^{*}\left(D_{\left(q^{-1}, q\right)}\right) g(x)\right]\right|_{x=0} .
$$

In this Bargmann-like representation, the $\left(q^{-1}, q\right)$-exponential function

$$
e_{\left(q^{-1}, q\right)}^{\alpha x}=\sum_{n=0}^{\infty} \frac{\alpha^{n} x^{n}}{[n]_{\left(q^{-1}, q\right)} !}
$$

for any complex number $\alpha$, is an eigenfunction of $a$, and

$$
\psi_{\alpha,\left(q^{-1}, q\right)}(x)=\frac{1}{\sqrt{e_{\left(q^{-1}, q\right)}^{|\alpha|^{2}}}} e_{\left(q^{-1}, q\right)}^{\alpha x}=\frac{1}{\sqrt{e_{\left(q^{-1}, q\right)}^{|\alpha|^{2}}}} \sum_{n=0}^{\infty} \frac{\alpha^{n}}{\sqrt{[n]_{\left(q^{-1}, q\right)} !}} \xi_{n,\left(q^{-1}, q\right)}(x)
$$


is a normalized coherent state of the $\left(q^{-1}, q\right)$-oscillator (63).

Representation theory of two-parameter quantum algebras led to the generalization of the $\left(q^{-1}, q\right)$-oscillator to the $(p, q)$-oscillator [40] (see also [41,42]):

$$
\begin{aligned}
{\left[N, a^{\dagger}\right] } & =a^{\dagger}, \quad[N, a]=-a, \quad\left[a, a^{\dagger}\right]=\phi(N+1)-\phi(N), \\
\phi(N) & =\frac{p^{N}-q^{N}}{p-q}=[N]_{(p, q)} .
\end{aligned}
$$

Since

$$
[N+1]_{(p, q)}-p[N]_{(p, q)}=q^{N}
$$

we can write

$$
a a^{\dagger}-p a^{\dagger} a=q^{N} .
$$

Note the $p \longleftrightarrow q$ symmetry. The Fock representation is

$$
\begin{aligned}
a^{\dagger}|n\rangle & =\sqrt{[n+1]_{(p, q)}}|n+1\rangle, \quad a|n\rangle=\sqrt{[n]_{(p, q)}}|n-1\rangle, \\
N|n\rangle & =n|n\rangle, \quad n=0,1,2, \ldots
\end{aligned}
$$

where

$$
[n]_{(p, q)}=\frac{p^{n}-q^{n}}{p-q}
$$

defines the $(p, q)$-basic number, or the twin-basic number [43]. Note that

$$
[0]_{(p, q)}=0, \quad[1]_{(p, q)}=1, \quad \lim _{p, q \longrightarrow 1}[n]_{(p, q)}=n .
$$

Now, with the definitions

$$
[0]_{(p, q)} !=1, \quad[n]_{(p, q)} !=\prod_{j=1}^{n}[j]_{(p, q)}, \quad n=1,2, \ldots
$$

we have

$$
|n\rangle=\frac{a^{\dagger^{n}}}{\sqrt{[n]_{(p, q)} !}}|0\rangle .
$$

Defining the $(p, q)$-exponential function as

$$
e_{(p, q)}^{x}=\sum_{n=0}^{\infty} \frac{x^{n}}{[n]_{(p, q)} !}
$$


the normalized coherent states of the $(p, q)$-oscillator (80) are given by

$$
|\alpha\rangle_{(p, q)}=\frac{1}{\sqrt{e_{(p, q)}^{|\alpha|^{2}}}} \sum_{n=0}^{\infty} \frac{\alpha^{n}}{\sqrt{[n]_{(p, q)} !}}|n\rangle=\frac{1}{\sqrt{e_{(p, q)}^{|\alpha|^{2}}}} e_{(p, q)}^{\alpha a^{\dagger}}|0\rangle .
$$

The Bargmann-like representation of the $(p, q)$-oscillator algebra (80) is given by

$$
a^{\dagger}=x, \quad a=D_{(p, q)}=\frac{1}{x}\left(\frac{p^{x D}-q^{x D}}{p-q}\right), \quad N=x D .
$$

The $(p, q)$-derivative $D_{(p, q)}$ is such that

$$
D_{(p, q)} F(x)=\frac{F(p x)-F(q x)}{(p-q) x}, \quad D_{(p, q)} x^{n}=[n]_{(p, q)} x^{n-1} .
$$

The monomials

$$
\xi_{n,(p, q)}(x)=\frac{x^{n}}{\sqrt{[n]_{(p, q)} !}}, \quad n=0,1,2, \ldots
$$

carry the Fock representation:

$$
\begin{aligned}
a^{\dagger} \xi_{n,(p, q)} & =\sqrt{[n+1]_{(p, q)}} \xi_{n+1,(p, q)}, \quad a \xi_{n,(p, q)}=\sqrt{[n]_{(p, q)}} \xi_{n-1,(p, q)}, \\
N \xi_{n,(p, q)} & =n \xi_{n,(p, q)}, \quad n=0,1,2, \ldots .
\end{aligned}
$$

The monomials $\left\{\xi_{n,(p, q)}(x) \mid n=0,1,2, \ldots\right\}$ form an orthonormal basis with respect to the inner product

$$
\langle f \mid g\rangle_{(p, q)}=\left.\left[f^{*}\left(D_{(p, q)}\right) g(x)\right]\right|_{x=0} .
$$

Note that, in this Bargmann-like representation, the $(p, q)$-exponential function

$$
e_{(p, q)}^{\alpha x}=\sum_{n=0}^{\infty} \frac{\alpha^{n} x^{n}}{[n]_{(p, q)} !}
$$

for any complex number $\alpha$, is an eigenfunction of $a$ and a normalized coherent state of the $(p, q)$-oscillator is given by

$$
\psi_{\alpha,(p, q)}(x)=\frac{1}{\sqrt{e_{(p, q)}^{|\alpha|^{2}}}} e_{(p, q)}^{\alpha x}=\frac{1}{\sqrt{e_{(p, q)}^{|\alpha|^{2}}}} \sum_{n=0}^{\infty} \frac{\alpha^{n}}{\sqrt{[n]_{(p, q)} !}} \xi_{n,(p, q)}(x) .
$$


Note that the canonical boson oscillator (13), the $q$-oscillator (45), and the $\left(q^{-1}, q\right)$-oscillator (63) , are special cases of the $(p, q)$-oscillator (80) corresponding to $(p=1, q=1), p=1$, and $p=q^{-1}$, respectively. The canonical fermion oscillator corresponds to $(p=-1, q=1)$. The $q$-fermion oscillator ( [44,45]) and the Tamm-Dancoff oscillator ( [46,47]) are also special cases of the $(p, q)$-oscillator corresponding to $p=-q^{-1}$ and $p=q$, respectively. Actually, one can choose $p$ as any function of $q$ in building models. Thus, starting with the $(p, q)$-oscillator a plethora of interesting types of $q$-deformed oscillators have been found with their energy spectra having accidental pairwise energy level degeneracies [48 50]. Further, there exist several other deformed oscillator structures with single and multiple deformation parameters (see, e.g., [51 55]). It may also be noted that $(p, q)$-calculus based on the $(p, q)$-number (84) and the $(p, q)$-derivative (91), sometimes called the post-quantum calculus following the name quantum calculus given to the calculus based on the $q$-number (49) and the $q$-derivative (56) (see [39]), is a very active area of research in applied mathematics with applications to approximation theory, computer-aided geometric design, etc. (see, e.g., [56 58] and references therein).

It is known since the early days of deformed oscillators that the creation and annihilation operators of the deformed oscillator (27) can be realized in terms of the canonical boson creation and annihilation operators as

$$
a^{\dagger}=\sqrt{\frac{\phi(N)}{N}} b^{\dagger}, \quad a=b \sqrt{\frac{\phi(N)}{N}}=\sqrt{\frac{\phi(N+1)}{N+1}} b, \quad N=b^{\dagger} b .
$$

Writing $\phi(N)=f^{2}(N) N$, the deformed oscillator (27) has been presented as

$$
\begin{aligned}
a^{\dagger} & =f(N) b^{\dagger}, \quad a=b f(N)=f(N+1) b, \quad N=b^{\dagger} b, \\
{\left[N, a^{\dagger}\right] } & =a^{\dagger}, \quad[N, a]=-a, \\
{\left[a, a^{\dagger}\right] } & =f^{2}(N+1)(N+1)-f^{2}(N) N,
\end{aligned}
$$

called an $f$-oscillator, and a general theory of $f$-oscillators has been developed [59] (see also [16, 17]) with many applications to nonlinear physics including nonlinear coherent states, or $f$-coherent states, relevant for quantum optics (see, e.g., 60]; see also 61 63]). The function $f(n)$, called the nonlinearity function, characterizes the $f$-oscillator (98). In terms of $f(n)$ the deformed exponential function (34) can be rewritten as

$$
e_{\phi}^{x}=\sum_{n=0}^{\infty} \frac{x^{n}}{f^{2}(n) ! n !}=e_{f}^{x}
$$


where

$$
f^{2}(0) !=1, \quad f^{2}(n) !=\prod_{j=1}^{n} f^{2}(j), \quad \text { for } n \geq 1 .
$$

Then, the normalized coherent states of the $f$-oscillator (98), eigenstates of $a=f(N+1) b$, are given by

$$
|\alpha\rangle_{f}=\frac{1}{\sqrt{e_{f}^{|\alpha|^{2}}}} \sum_{n=0}^{\infty} \frac{\alpha^{n}}{f(n) ! \sqrt{n !}}|n\rangle,
$$

where $f(0) !=1, f(n) !=\prod_{j=1}^{n} f(j)$ for $n \geq 1$, and $\alpha$ is such that $e_{f}^{|\alpha|^{2}}<\infty$. These states (101) are called nonlinear coherent states, or $f$-coherent states, of the boson oscillator and are characterized by the nonlinearity function $f(n)$. They correspond to the nonlinear coherent states of photons in the context of quantum optics. When $f(n)=1 / \sqrt{n}$ the $f$-coherent state (101) becomes

$$
|\alpha\rangle_{h}=\sqrt{1-|\alpha|^{2}} \sum_{n=0}^{\infty} \alpha^{n}|n\rangle, \quad \text { where }|\alpha|<1,
$$

known variously as phase coherent state [64, harmonious state [65], or pseudothermal state [66] (see, e.g., 67] for more details).

\section{Tsallis q-exponential function as a $\phi$ - exponential function}

It has been shown in [68] that expansion of the Tsallis q-exponential function in Taylor series leads to the expression

$$
\begin{aligned}
e_{q}(x) & =1+\sum_{n=1}^{\infty} \frac{Q_{n-1}}{n !} x^{n} \\
Q_{0} & =1, \quad Q_{n}=q(2 q-1)(3 q-2) \ldots(n q-(n-1)), \quad n=1,2, .
\end{aligned}
$$

Writing the expression for $e_{f}^{x}$ in (99) as

$$
e_{f}^{x}=1+\sum_{n=1}^{\infty} \frac{x^{n}}{f^{2}(n) ! n !}
$$


and comparing this expression with (103) one identifies $e_{q}(x)$ with $e_{f}^{x}$ corresponding to

$$
f(0) !=1, \quad f(n) !=\frac{1}{\sqrt{Q_{n-1}}}, \quad n=1,2, \ldots
$$

In [69] this choice of $f(n)$ ! has been substituted in (101) to get the normalized states

$$
\begin{aligned}
|\alpha\rangle & =\mathcal{N}(\alpha)\left\{|0\rangle+\sum_{n=1}^{\infty} \sqrt{Q_{n-1}} \frac{\alpha^{n}}{\sqrt{n !}}|n\rangle\right\}, \\
\mathcal{N}(\alpha) & =\frac{1}{\sqrt{1+\sum_{n=1}^{\infty} Q_{n-1} \frac{|\alpha|^{2}}{n !}}},
\end{aligned}
$$

called the $f$-coherent states attached to the Tsallis $q$-exponential function. This class of $f$-coherent states and their nonclassical properties and other physical aspects have been studied in detail for $1<q \leq 2$ in [69].

Noting that in (103)

$$
Q_{n-1}=(1+(q-1)(n-1)) !=\prod_{j=1}^{n}(1+(q-1)(j-1)), \quad n=1,2, \ldots,
$$

the Taylor series expression for $e_{q}(x)$ has been identified in [70] with a deformed exponential function as

$$
e_{q}(x)=\sum_{n=0}^{\infty} \frac{x^{n}}{[n]_{(q-1)} !}
$$

where

$$
\begin{aligned}
{[n]_{(q-1)} } & =\frac{n}{1+(q-1)(n-1)}, \quad n=0,1,2, \ldots \\
{[0]_{q-1} ! } & =1, \quad[n]_{(q-1)} !=\prod_{j=1}^{n}[j]_{(q-1)}=\frac{n !}{(1+(q-1)(n-1)) !}, \text { for } n \geq 1 .
\end{aligned}
$$

Note that

$$
[0]_{(q-1)}=0, \quad[1]_{(q-1)}=1, \quad \lim _{q \longrightarrow 1}[n]_{(q-1)}=n .
$$


Regarding $[n]_{(q-1)}$ as a deformed number, a scheme of $q$-deformation of nonlinear maps was proposed in [70] and this proposal has been found to have many applications (see, e.g., [71 76]). Equation (108) shows that we can write $e_{q}(x)$ as a $\phi$-exponential function:

$$
e_{q}(x)=\sum_{n=0}^{\infty} \frac{x^{n}}{\phi_{T}(n) !}=e_{\phi_{T}}^{x}, \text { with } \phi_{T}(n)=[n]_{(q-1)} .
$$

Let us close this section with an interesting observation. Note that we can write

$$
e_{q}(x)=e^{\ln \left((1+(1-q) x)^{\frac{1}{1-q}}\right)}=e^{\sum_{n=1}^{\infty} \frac{(q-1)^{n-1}}{n} x^{n}},
$$

showing explicitly $\lim _{q \longrightarrow 1} e_{q}(x)=e^{x}$. The expression (112) for $e_{q}(x)$ as an ordinary exponential could be useful for approximations in applications. In particular, for the Tsallis $q$-Gaussian function we can write, for $q \approx 1$,

$$
e_{q}\left(-\beta x^{2}\right) \approx e^{-\beta x^{2}+\frac{q-1}{2} \beta^{2} x^{4}-\frac{(q-1)^{2}}{3} \beta^{3} x^{6}} .
$$

In [77] (see also [78]) it has been shown that if

$$
h(x)=\sum_{n=1}^{\infty} a_{n} x^{n}
$$

then

$$
e^{h(x)}=\sum_{n=0}^{\infty} c_{n} x^{n}
$$

where $c_{0}=1, c_{1}=a_{1}$, and

$$
c_{n}=a_{n}+\frac{1}{n} \sum_{j=1}^{n-1} j c_{n-j} a_{j}, \quad n=2,3, \ldots .
$$

Using this result the $q$-exponential function $e_{q}^{x}$ in (53) has been expressed as the exponential of an infinite series in [79]. For the Tsallis $q$-exponential function we have

$$
\begin{aligned}
h(x) & =\sum_{n=1}^{\infty} \frac{(q-1)^{n-1}}{n} x^{n}, \\
e^{h(x)} & =e_{q}(x)=\sum_{n=0}^{\infty} \frac{x^{n}}{[n]_{(q-1)} !}=1+x+\sum_{n=2}^{\infty} \frac{(1+(q-1)(n-1)) !}{n !} x^{n} .
\end{aligned}
$$


We find $c_{0}=1$ and $c_{1}=a_{1}=1$ as should be. Now, substituting

$$
a_{n}=\frac{(q-1)^{n-1}}{n}, \quad c_{n}=\frac{(1+(q-1)(n-1)) !}{n !}, \quad \text { for } n \geq 2,
$$

in (116) we get an identity: for $n \geq 2$,

$$
\begin{aligned}
& \frac{(1+(n-1)(q-1)) !}{n !} \\
& \quad=\frac{(q-1)^{n-1}}{n}+\frac{1}{n} \sum_{j=1}^{n-1} \frac{(q-1)^{j-1}(1+(n-j-1)(q-1)) !}{(n-j) !} .
\end{aligned}
$$

We can verify this identity directly. Let us take $(q-1)=1 / \tau$. Then the identity (119) becomes

$$
(\tau)_{n}=(n-1) ! \tau \sum_{j=0}^{n-1} \frac{(\tau)_{j}}{j !}
$$

where $(\tau)_{n}=\tau(\tau+1)(\tau+2) \ldots(\tau+(n-1))$ is the rising factorial, or the Pochhammer symbol, with $(\tau)_{0}=1$. Now, observe that

$$
(\tau)_{n+1}=n ! \tau \sum_{j=0}^{n} \frac{(\tau)_{j}}{j !}=n\left[(n-1) ! \tau\left(\frac{(\tau)_{n}}{n !}+\sum_{j=0}^{n-1} \frac{(\tau)_{j}}{j !}\right)\right]=(\tau+n)(\tau)_{n}
$$

showing that $(\tau)_{n}$ given by the right hand side of (120) satisfies the defining recurrence relation for $(\tau)_{n}$, namely, $(\tau)_{n+1}=(\tau+n)(\tau)_{n}$ with $(\tau)_{0}=1$.

As observed in [80], we can represent the Tsallis $q$-exponential function as a hypergeometric series (see, e.g., [81] for details of the theory of hypergeometric series) as follows:

$$
e_{q}(x)={ }_{1} F_{0}\left(\frac{1}{q-1} ;-;(q-1) x\right)
$$

where

$$
{ }_{1} F_{0}(a ;-; z)=\sum_{n=0}^{\infty}(a)_{n} \frac{z^{n}}{n !}
$$


This relation can be verified as follows:

$$
\begin{aligned}
e_{q}(x) & =\sum_{n=0}^{\infty} \frac{x^{n}}{[n]_{q-1} !}=1+\sum_{n=1}^{\infty}\left(\prod_{j=1}^{n}(1+(q-1)(j-1))\right) \frac{x^{n}}{n !} \\
& =1+\sum_{n=1}^{\infty}\left(\prod_{j=1}^{n}\left(\frac{1}{q-1}+(j-1)\right)\right) \frac{((q-1) x)^{n}}{n !} \\
& =\sum_{n=0}^{\infty}\left(\frac{1}{q-1}\right)_{n} \frac{((q-1) x)^{n}}{n !} \\
& ={ }_{1} F_{0}\left(\frac{1}{q-1} ;-;(q-1) x\right) .
\end{aligned}
$$

One may also identify $e_{q}(x)$ with ${ }_{2} F_{1}\left(\frac{1}{q-1}, b ; b ;(q-1) x\right)$, where $b$ is an arbitrary nonzero parameter, since ${ }_{1} F_{0}(a ;-; x)={ }_{2} F_{1}(a, b ; b ; x)$, where

$$
{ }_{2} F_{1}(a, b ; c ; z)=\sum_{n=0}^{\infty} \frac{(a)_{n}(b)_{n}}{(c)_{n}} \frac{z^{n}}{n !}
$$

is the Gauss hypergeometric function.

For the Tsallis $q$-logarithm we get

$$
\ln _{q}(1+x)=x_{2} F_{1}(q, 1 ; 2 ;-x) .
$$

The proof of this relation is as follows:

$$
\begin{aligned}
\ln _{q}(1+x) & =\frac{(1+x)^{1-q}-1}{1-q}=\frac{1}{1-q}\left(\sum_{n=0}^{\infty}\left(\begin{array}{c}
1-q \\
n
\end{array}\right) x^{n}-1\right) \\
& =x \sum_{n=0}^{\infty}(q)_{n} \frac{(-x)^{n}}{(n+1) !}=x \sum_{n=0}^{\infty} \frac{(q)_{n}(1)_{n}}{(2)_{n}} \frac{(-x)^{n}}{n !} \\
& =x{ }_{2} F_{1}(q, 1 ; 2 ;-x) .
\end{aligned}
$$

Using the well known Euler integral representation of ${ }_{2} F_{1}(a, b ; c ; z)$ it can be verified that

$$
x_{2} F_{1}(q, 1 ; 2 ;-x)=\int_{0}^{1} d t \frac{x}{(1+x t)^{q}}=\frac{(1+x)^{1-q}-1}{1-q}=\ln _{q}(1+x) .
$$

Note that when $q \longrightarrow 1$ the relation (126) becomes the well known relation $\ln (1+x)=x_{2} F_{1}(1,1 ; 2 ;-x)$. We hope these identifications would help further analysis and generalizations of the Tsallis $q$-exponential and $q$-logarithm functions. 


\section{The deformed oscillator and the deformed derivative associated with the Tsallis $q-$ exponential function}

Let us now consider the deformed oscillator with the commutation relations

$$
\begin{aligned}
{\left[N, a^{\dagger}\right] } & =a^{\dagger}, \quad[N, a]=-a, \quad\left[a, a^{\dagger}\right]=\phi_{T}(N+1)-\phi_{T}(N), \\
\phi_{T}(N) & =[N]_{(q-1)}=\frac{N}{1+(q-1)(N-1)} .
\end{aligned}
$$

The energy spectrum of this deformed oscillator is given by

$$
\begin{aligned}
E_{n, T} & =\frac{1}{2}\left(\phi_{T}(n+1)+\phi_{T}(n)\right) \\
& =\frac{1}{2}\left(\frac{2(q-1) n^{2}+2 n+2-q}{(q-1)^{2} n^{2}+(3-q)(q-1) n+2-q}\right), \\
& \quad n=0,1,2, \ldots,
\end{aligned}
$$

as follows from (32). For $1 \leq q \leq 2$ we find that

$$
\begin{aligned}
E_{n, T}(q=1) & =n+\frac{1}{2}, \quad n=0,1,2, \ldots, \\
E_{0, T} & =\frac{1}{2}, \quad \text { for } 1 \leq q<2, \quad E_{0, T}=\frac{1}{2}, \quad \text { when } q \longrightarrow 2, \\
E_{n, T} & =1, \text { for all } n>0, \quad \text { when } q \stackrel{1}{\longrightarrow}, \\
\lim _{n \longrightarrow \infty} E_{n, T} & =\frac{1}{q-1}, \quad \text { for } 1 \leq q \leq 2, \\
E_{n+1, T}-E_{n, T} & =\frac{2-q}{(q-1)^{2} n^{2}+2(q-1) n+q(2-q)} \geq 0 \\
\lim _{q \longrightarrow 2}\left(E_{n+1, T}-E_{n, T}\right) & =0, \text { for all } n>0 .
\end{aligned}
$$

From these equations we can conclude as follows. The deformed oscillator (129) becomes a canonical boson oscillator when $q=1$. For $1<q \leq 2$ it has a ground state with energy $E_{0, T}=1 / 2$, same as a boson, and an infinity of excited states starting with $E_{1, T}=(1 / 2)+(1 / q)$ and increasing in diminishing steps up to the maximum energy $E_{\infty, T}=1 /(q-1)<\infty$. As the value of $q$ increases from 1 to 2 the width of this band of energies of excited 
states, $E_{\infty, T}-E_{1, T}=(1 /(q(q-1)))-(1 / 2)$, decreases and when $q \longrightarrow 2$ the energy band shrinks to a single energy level with infinite degeneracy $\left(E_{n, T}=1\right.$ for all $\left.n>0\right)$. Thus, when $q \longrightarrow 2$ this oscillator becomes a twolevel system with a nondegenerate ground state with energy $E_{0, T}=1 / 2$ and an infinitely degenerate excited state with energy eigenvalues $E_{n, T}=1$ for all $n>0$. It should be interesting to investigate the physical applications of such deformed oscillators related to the Tsallis $q$-exponential function. The deformed oscillator algebra (129) has been derived in [82 in a completely different context, unrelated to the Tsallis $q$-exponential function, and the thermodynamical properties of a gas of photons obeying such an algebra have been evaluated (see also [20] for a discussion of the algebra (129)).

It is seen that the $\mu$-oscillator, introduced in 83 , for which $\phi(N)=$ $N /(1+\mu N)$, with $\mu>0$, is only slightly different from (129). The $\mu$-oscillator is the first deformed oscillator found with its energies lying within a band of finite width exactly like in the case of the deformed oscillator (129). For $\mu=0$ the $\mu$-oscillator becomes a canonical boson oscillator and for any $\mu>0$ it has a spectrum bounded from above, but it does not share the other interesting properties of the deformed oscillator (129). This can be seen by comparing (131) with the energy spectrum of the $\mu$-oscillator:

$$
\begin{aligned}
E_{n, \mu} & =\frac{1}{2}\left(\frac{n+1}{1+\mu(n+1)}+\frac{n}{1+\mu n}\right) \\
& =\frac{1}{2}\left(\frac{2 \mu n^{2}+2(\mu+1) n+1}{\mu^{2} n^{2}+2 \mu n+\mu^{2}+\mu+1}\right), \\
E_{n, \mu=0} & =n+\frac{1}{2}, \quad n=0,1,2, \ldots, \\
E_{0, \mu} & =\frac{1}{2\left(\mu^{2}+\mu+1\right)}, \text { for } \mu \geq 0, \\
\lim _{n \rightarrow \infty} E_{n, \mu} & =\frac{1}{\mu}, \text { for } \mu>0, \\
E_{n+1, \mu}-E_{n, \mu} & =\frac{1}{\mu^{2} n^{2}+2 \mu(\mu+1) n+2 \mu+1}, \text { for } \mu \geq 0 .
\end{aligned}
$$

In particular, note that there is no finite value of $\mu$ for which $E_{n, \mu}$ becomes independent of $n$ for all $n>0$. It is this property which makes the deformed oscillator (129) become a two-level system when $q \longrightarrow 2$.

The deformed oscillator (129) can be presented as an $f$-oscillator as fol- 
lows:

$$
\begin{aligned}
a^{\dagger} & =f_{T}(N) b^{\dagger}, \quad a=b f_{T}(N)=f_{T}(N+1) b, \quad N=b^{\dagger} b, \\
f_{T}(N) & =\sqrt{\frac{\phi_{T}(N)}{N}}=\sqrt{\frac{[N]_{(q-1)}}{N}}=\frac{1}{\sqrt{(1+(q-1)(N-1)))}} .
\end{aligned}
$$

It has been found in [69] that the states in (106) are the coherent states of this $f$-oscillator corresponding to the eigenfunctions of $a=f_{T}(N+1) b$ and are related to the Tsallis $q$-exponential function. To understand this let us proceed as follows. The Fock representation of (129) is given by

$$
\begin{aligned}
a^{\dagger}|n\rangle & =\sqrt{[n+1]_{(q-1)}}|n+1\rangle, \quad a|n\rangle=\sqrt{[n]_{(q-1)}}|n-1\rangle, \\
N|n\rangle & =n|n\rangle, \quad n=0,1,2, \ldots .
\end{aligned}
$$

It follows that

$$
|n\rangle=\frac{a^{\dagger^{n}}}{\sqrt{[n]_{(q-1) !}}}|0\rangle .
$$

With the Tsallis $q$-exponential function expressed as in (108), the normalized coherent states of the deformed oscillator (129) are seen to be given by

$$
|\alpha\rangle_{T}=\frac{1}{\sqrt{e_{q}\left(|\alpha|^{2}\right)}} \sum_{n=0}^{\infty} \frac{\alpha^{n}}{\sqrt{[n]_{(q-1)} !}}|n\rangle=e_{q}\left(\alpha a^{\dagger}\right)|0\rangle .
$$

Noting that

$$
|n\rangle=\frac{a^{\dagger^{n}}}{\sqrt{[n]_{(q-1)} !}}|0\rangle=\frac{\left(f_{T}(N) b^{\dagger}\right)^{n}}{\sqrt{[n]_{(q-1)} !}}|0\rangle=\frac{f_{T}(n) ! b^{\dagger^{n}}}{\sqrt{[n]_{(q-1)} !}}|0\rangle,
$$

and using (99), (101), and (111), it is seen that

$$
|\alpha\rangle_{T}=\frac{1}{\sqrt{e_{q}\left(|\alpha|^{2}\right)}} \sum_{n=0}^{\infty} \frac{f_{T}(n) ! \sqrt{n !} \alpha^{n}}{[n]_{(q-1)} !}|n\rangle=\frac{1}{\sqrt{e_{f_{T}}^{|\alpha|^{2}}}} \sum_{n=0}^{\infty} \frac{\alpha^{n}}{f_{T}(n) ! \sqrt{n !}}|n\rangle,
$$

is an $f$-coherent state of the boson oscillator associated with the Tsallis $q$ exponential function as identified in [69].

To get the Bargmann-like representation of the deformed oscillator (129) we must have a deformed derivative $D_{(T, q)}$ such that

$$
D_{(T, q)} x^{n}=[n]_{(q-1)} x^{n-1} .
$$


Following (39), as has been done in $([82,84,85])$, one may choose the formal operator $(1 / x) \phi(x D)=(1 /(1+(q-1) x D)) D$ as the required deformed derivative which satisfies (139) and has $e_{q}(k x)$ as its eigenfunction for any $k$. However, in this case it is only a formal operator, not suitable to operate on an arbitrary function without a series expansion. Another suggestion for the required deformed derivative is $(1+(1-q) x) D$ for which $e_{q}(x)$ is an eigenfunction (see [86, 87]). But, it is also not suitable for the purpose since $e_{q}(k x)$ is not its eigenfunction for arbitrary $k$ unless one defines $e_{q}(k x)=\left(e_{q}(x)\right)^{k}$ as in [34, 35, 80]. As we have seen earlier, for the Tsallis $q$-exponential function $e_{q}(k x)=(1+(1-q) k x)^{1 /(1-q)} \neq e_{q}(x)^{k}$. Further, the derivative $(1+(1-q) x) D$ does not satisfy the requirement in (139).

To derive the required deformed derivative we follow [88] where the deformed derivative corresponding to the $\mu$-oscillator [83] has been obtained. Slightly modifying the suggestion in [88] we get the desired deformed derivative as

$$
D_{(T, q)} F(x)=\int_{0}^{1} d t t^{1-q} D F\left(t^{q-1} x\right) .
$$

It can be verified that for $F(x)=x^{n}$

$$
\begin{aligned}
D_{(T, q)} x^{n} & =\int_{0}^{1} d t t^{1-q} D\left(t^{(q-1) n} x^{n}\right)=\int_{0}^{1} d t t^{(q-1)(n-1)} D x^{n} \\
& =n x^{n-1} \int_{0}^{1} d t t^{(q-1)(n-1)}=\frac{n x^{n-1}}{1+(q-1)(n-1)} \\
& =[n]_{(q-1)} x^{n-1},
\end{aligned}
$$

as required. Then, it follows that

$$
D_{(T, q)} e_{q}(k x)=D_{(T, q)}\left(\sum_{n=0}^{\infty} \frac{k^{n} x^{n}}{[n]_{(q-1)} !}\right)=k e_{q}(k x) .
$$

It can also be verified directly that

$$
D_{(T, q)} e_{q}(k x)=k e_{q}(k x) .
$$

This is seen as follows. From (140) we have

$$
D_{(T, q)} e_{q}(k x)=\int_{0}^{1} d t t^{1-q} D\left[\left(1+(1-q) k t^{q-1} x\right)^{\frac{1}{1-q}}\right]
$$




$$
\begin{aligned}
& =k \int_{0}^{1} d t\left(1+(1-q) k t^{q-1} x\right)^{\frac{q}{1-q}} \\
& =k \int_{0}^{1} d t t^{-q}\left(t^{1-q}+(1-q) k x\right)^{\frac{q}{1-q}} \\
& =\left.\left[\frac{k}{1-q} \int d\left(t^{1-q}\right)\left(t^{1-q}+(1-q) k x\right)^{\frac{q}{1-q}}\right]\right|_{t=0} ^{t=1} \\
& =\left.\left[k t\left(1+(1-q) k t^{q-1} x\right)^{\frac{1}{1-q}}\right]\right|_{t=0} ^{t=1} \\
& =\left.\left[k t e_{q}\left(k t^{q-1} x\right)\right]\right|_{t=0} ^{t=1}=k e_{q}(k x),
\end{aligned}
$$

proving (143). The inverse of the deformed derivative $D_{(T, q)}$, the deformed integral, is seen to be given by

$$
\int d_{(T, q)} x f(x)=\left.\left[\frac{d}{d t}\left(\int d x t f\left(t^{(q-1)} x\right)\right)\right]\right|_{t=0} ^{t=1}
$$

such that if $\int d_{(T, q)} x f(x)=F(x)$ then $D_{T, q} F(x)=f(x)$. It can be verified that

$$
\int d_{(T, q)} x x^{n}=\left.\left[\frac{d}{d t}\left(\int d x t^{(q-1) n+1} x^{n}\right)\right]\right|_{t=0} ^{t=1}=\frac{x^{n+1}}{[n+1]_{(q-1)}}
$$

as should be, since $D_{(T, q)} x^{n+1}=[n+1]_{(q-1)} x^{n}$.

Now, the Bargmann-like representation of the deformed oscillator (129) is given by

$$
a^{\dagger}=x, \quad a=D_{(T, q)}, \quad N=x D .
$$

Under the inner product

$$
\langle f \mid g\rangle_{T}=\left.\left[f^{*}\left(D_{(T, q)}\right) g(x)\right]\right|_{x=0},
$$

the monomials

$$
\xi_{n, T}(x)=\frac{x^{n}}{\sqrt{[n]_{(q-1)} !}}, \quad n=0,1,2, \ldots
$$

form an orthonormal basis and carry the Fock representation

$$
\begin{aligned}
& a^{\dagger} \xi_{n, T}(x)=\sqrt{[n+1]_{(q-1)}} \xi_{n+1, T}(x), \quad a \xi_{n, T}(x)=\sqrt{[n]_{(q-1)}} \xi_{n-1, T}(x), \\
& N \xi_{n, T}(x)=n \xi_{n, T}(x), \quad n=0,1,2, \ldots .
\end{aligned}
$$


In the Bargmann-like representation, with

$$
\left\langle e_{q}(\alpha x) \mid e_{q}(\alpha x)\right\rangle_{T}=e_{q}\left(|\alpha|^{2}\right),
$$

the normalized coherent states of the deformed oscillator (129), eigenfunctions of $a=D_{(T, q)}$, are given by

$$
\begin{aligned}
\psi_{\alpha, T}(x) & =\frac{1}{\sqrt{e_{q}\left(|\alpha|^{2}\right)}} e_{q}(\alpha x)=\frac{1}{\sqrt{e_{q}\left(|\alpha|^{2}\right)}} \sum_{n=0}^{\infty} \frac{\alpha^{n} x^{n}}{[n]_{(q-1)} !} \\
& =\frac{1}{\sqrt{e_{q}\left(|\alpha|^{2}\right)}} \sum_{n=0}^{\infty} \frac{\alpha^{n}}{\sqrt{[n]_{(q-1)} !}} \xi_{n, T}(x) .
\end{aligned}
$$

The case $q=2$ is interesting. In this case we have $f_{T}(n)=1 / \sqrt{n}$ and the state $|\alpha\rangle_{T}$ becomes the harmonious state (102) as observed in [69]. Now, for $q=2$ we have

$$
[0]_{(1)}=0, \quad[n]_{(1)}=1, \text { for all } n .
$$

Thus, for $q=2$ the deformed oscillator (129) has the Fock representation

$$
\begin{aligned}
a^{\dagger}|n\rangle & =|n+1\rangle, \quad n=0,1,2, \ldots, \\
a|0\rangle & =0, \quad a|n\rangle=|n-1\rangle, \quad n=1,2, \ldots, \\
N|n\rangle & =n|n\rangle, \quad n=0,1,2, \ldots,
\end{aligned}
$$

and can be identified with the algebra of the Susskind-Glogower exponential phase operators (see, e.g., [59]). The coherent states of this deformed oscillator are

$$
|\alpha\rangle_{(T, q=2)}=\sqrt{1-|\alpha|^{2}} \sum_{n=0}^{\infty} \alpha^{n}|n\rangle, \quad|\alpha|<1,
$$

where $\sqrt{1-|\alpha|^{2}}=1 / \sqrt{e_{2}\left(|\alpha|^{2}\right)}$ in which $e_{2}\left(|\alpha|^{2}\right)$ is a Tsallis $q$-exponential function corresponding to $q=2$.

It is instructive to look at the deformed derivative and integral operators explicitly for $q=2$. Corresponding to $q=2$ the deformed derivative becomes, as seen from (140),

$$
D_{(T, q=2)} F(x)=\int_{0}^{1} d t t^{-1} D F(t x) .
$$


For $F(x)=x^{n}$

$$
D_{(T, q=2)} x^{n}=\int_{0}^{1} d t n t^{n-1} x^{n-1}=x^{n-1},
$$

as required. To verify that

$$
e_{2}(k x)=\frac{1}{1-k x}
$$

is an eigenfunction of $D_{(T, q=2)}$, satisfying

$$
D_{(T, q=2)} e_{2}(k x)=k e_{2}(k x),
$$

we follow (144) to get

$$
\begin{aligned}
D_{(T, q=2)} e_{2}(k x) & =\int_{0}^{1} d t t^{-1} D\left[(1-k t x)^{-1}\right] \\
& =k \int_{0}^{1} d t(1-k t x)^{-2} \\
& =k \int_{0}^{1} d t t^{-2}\left(t^{-1}-k x\right)^{-2} \\
& =\left.\left[-k \int d\left(t^{-1}\right)\left(t^{-1}-k x\right)^{-2}\right]\right|_{t=0} ^{t=1} \\
& =\left.\left[k t(1-k t x)^{-1}\right]\right|_{t=0} ^{t=1} \\
& =\left.\left[k t e_{2}(k t x)\right]\right|_{t=0} ^{t=1}=k e_{2}(k x) .
\end{aligned}
$$

In this case the deformed integral is, as seen from (145),

$$
\int d_{(T, q=2)} x f(x)=\left.\left[\frac{d}{d t}\left(\int d x t f(t x)\right)\right]\right|_{t=0} ^{t=1} .
$$

For $f(x)=x^{n}$

$$
\int d_{(T, q=2)} x x^{n}=\left.\left[\frac{d}{d t}\left(\int d x t^{n+1} x^{n}\right)\right]\right|_{t=0} ^{t=1}=x^{n+1}
$$

as should be, since $D_{(T, q=2)} x^{n+1}=x^{n}$. 


\section{Conclusion}

In fine, it is found that the deformed oscillator defined by the commutation relations

$$
\begin{aligned}
{\left[N, a^{\dagger}\right]=} & a^{\dagger}, \quad[N, a]=-a \\
{\left[a, a^{\dagger}\right]=} & \phi_{T}(N+1)-\phi_{T}(N) \\
& \quad \text { with } \phi_{T}(N)=\frac{N}{1+(q-1)(N-1)}
\end{aligned}
$$

is associated with the Tsallis q-exponential function

$$
e_{q}(x)=(1+(1-q) x)^{\frac{1}{1-q}}
$$

representable as

$$
e_{q}(x)=\sum_{n=0}^{\infty} \frac{x^{n}}{\phi_{T}(n) !}, \quad \text { with } \phi_{T}(n) !=\phi_{T}(n) \phi_{T}(n-1) \ldots \phi_{T}(2) \phi_{T}(1) .
$$

In a Bargmann-like representation the annihilation operator $a$ corresponds to a deformed derivative defined by

$$
D_{(T, q)} F(x)=\int_{0}^{1} d t t^{1-q} D F\left(t^{q-1} x\right)
$$

with the Tsallis $q$-exponential functions as its eigenfunctions. Thus the Tsallis $q$-exponential functions are the coherent states of the deformed oscillator (129). When $q=2$ these deformed oscillator coherent states correspond to states known variously as phase coherent states, harmonious states, or pseudothermal states. Further, when $q=1$ this deformed oscillator is a canonical boson oscillator, when $1<q<2$ it has an energy spectrum bounded from above and with the ground state same as a boson, and when $q \longrightarrow 2$ it becomes a two-level system with a nondegenerate ground state and an infinitely degenerate excited state. It should be worthwhile to study the physical applications of such deformed oscillators. The expression in (166) for the deformed derivative $D_{(T, q)}$ for which the Tsallis $q$-exponential functions are eigenfunctions should lead to interesting applications, particularly, in nonextensive statistical mechanics which is essentially based on the Tsallis $q$-exponential function and its inverse function, namely, the Tsallis $q$-logarithm function. A 
remark in [88], made in the context of the $\mu$-oscillator, points to the existence of possibilities for extending the deformed derivative (166) and the Tsallis $q$-exponential function with more deformation parameters. For example, following the remark in [88], if we substitute the ordinary derivative $D$ in (166) by the two-parameter derivative $D_{(p, r)}\left(D_{(p, q)}\right.$ in (91) with $q$ replaced by $\left.r\right)$ we would get a $(p, q, r)$-deformed derivative and its eigenfunctions would be the Tsallis $(p, q, r)$-exponential functions (see [89] for the existing two-parameter generalization of the Tsallis $q$-logarithm and $q$-exponential function).

\section{References}

[1] Sternheimer, D., Deformation theory and physics model building, in Topics in Mathematical Physics, General Relativity, and Cosmology in Honor of Jerzy Plebański, Eds. H. García-Compeán, B. Mielnik, M. Montesnos, and M. Przanowski, 469-487, (World Scientific, 2006). https://doi.org/10.1142/9789812772732_0039.

[2] Curtright, T., Fairlie, D., and Zachos, C. (Eds.), Quantum Groups: Proceedings of the Argonne Workshop, (World Scientific, 1991). https://www.worldscientific.com/worldscibooks/10.1142/1206.

[3] Tsallis, C., Possible generalization of BoltzmannGibbs statistics, J. Stat. Phys. $52 \quad 479-487 \quad$ (1988). https://doi.org/10.1007/BF01016429.

[4] Arik, M. and Coon, D. D., Hilbert spaces of analytic functions and generalized coherent states, J. Math. Phys. 17 524-527 (1976). https://doi.org/10.1063/1.522937.

[5] Kuryshkin, V. V., Generalized quantum operators of creation and annihilation, Ann. Fond. L. de Broglie 5 111-125 (1980). https://inis. iaea.org/search/searchsinglerecord. aspx?recordsFor=SingleRecord\&RN=1

[6] Jannussis, A., Brodimas, G., Sourlas, D., and Zisis, V., Remarks on the q-quantization, Lett. Nuovo Cimento 30 123-127 (1981). https://doi.org/10.1007/BF02817324.

[7] Mansour, T. and Schork, M. Commutation Relations, Normal Ordering, and Stirling Numbers, (Taylor \& Francis, 2016). https://doi.org/10.1201/b18869. 
[8] Macfarlane, A. J., On $q$-analogues of the quantum harmonic oscillator and the quantum group $S U(2)_{q}$, J. Phys. A: Math. Gen. 22 4581-4588 (1989). https://doi.org/10.1088/0305-4470/22/21/020.

[9] Biedenharn, L. C., The quantum group $S U_{q}(2)$ and a $q$-analogue of the boson operators, J. Phys. A: Math. Gen. 22 L873-L878 (1989). https://doi.org/10.1088/0305-4470/22/18/004.

[10] Sun, C. P. and Fu, H. C., The q-deformed boson realisation of the quantum group $S U(n)_{q}$, and its representations, J. Phys. A: Math. Gen. 22 L983-L986 (1989). https://doi.org/10.1088/0305-4470/22/21/001.

[11] Hayashi, T., $Q$-analogues of Clifford and Weyl algebras-spinor and oscillator representations of quantum enveloping algebras, Commun. Math. Phys. 127 129-144 (1990). https://doi.org/10.1007/BF02096497.

[12] Bonatsos, D. and Daskaloyannis, C., Quantum groups and their applications in nuclear physics, Prog. Part. Nucl. Phys. 44 537-618 (1999). https://doi.org/10.1016/S0146-6410(99)00100-3

[13] Raychev, P. P., Quantum Groups: Application to Nuclear and Molecular Spectroscopy, in Advances in Quantum Chemistry, 26 239357, Eds. J. R. Sabin and M. C. Zerner, (Academic Press, 1995). https://doi.org/10.1016/S0065-3276(08)60114-8.

[14] Chaichian, M. and Demichev, A., Introduction to Quantum Groups, (World Scientific, 1996). https://doi.org/10.1142/3065.

[15] Chaichian, M., Felipe, R. G., and Montonen, C., Statistics of $q$-oscillators, quons, and relation to fractional statistics, J. Phys. A: Math. Gen. $26 \quad 4017-4034$ (1993). https://doi.org/10.1088/0305-4470/26/16/018.

[16] Shanta, P., Chaturvedi, S., Srinivasan, V., and Jagannathan, R., Unified approach to the analogues of single-photon and multiphoton coherent states for generalized bosonic oscillators, J. Phys. A: Math. Gen. 27 6433-6442 (1994). https://doi.org/10.1088/0305-4470/27/19/016.

[17] Sunilkumar, V., Bambah, B. A., Jagannathan, R., Panigrahi, P. K., and Srinivasan, V., Coherent states of nonlinear algebras: Applications to 
quantum optics, J. Opt. B: Quantum Semiclass. Opt., 2 126-132 (2000). https://doi.org/10.1088/1464-4266/2/2/311.

[18] Lavagno, A. and Narayana Swamy, P., Generalized thermodynamics of $q$-deformed bosons and fermions, Phys. Rev. E 65036101 (2002). https://doi.org/10.1103/PhysRevE.65.036101.

[19] Narayana Swamy, P., Interpolating statistics and qoscillator algebras, Int. J. Mod. Phys. B 20 697-713 (2006). https://doi.org/10.1142/S0217979206033498.

[20] Chung, W. S. and Algin, A., Modified multidimensional qdeformed Newton oscillators: Algebra, interpolating statistics, and thermodynamics, Annal. Phys. 409167911 (2019). https://doi.org/10.1016/j.aop.2019.167911

[21] Marinho, A., Brito, F. A., and Chesman, C., Thermal properties of a solid through q-deformed algebra, Physica A 391 3424-3434 (2012). https://doi.org/10.1016/j.physa.2012.02.012

[22] Tristant, D. and Brito, F. A., Some electronic properties of metals through q-deformed algebra, Physica A 407 276-286 (2014). https://doi.org/10.1016/j.physa.2014.04.008.

[23] Haghshenasfard, Z. and Cottam, M. G., $q$-deformed model of nonclassical quantum-statistical behaviour for an atom laser, Euro. Phys. J. D 67195 (2013). https://doi.org/10.1140/epjd/e2013-40159-5.

[24] Dey, S., $q$-deformed noncommutative cat states and their nonclassical properties, Phys. Rev. D 91044024 (2015). https://doi.org/10.1103/PhysRevD.91.044024.

[25] Dey, S. and Hussin, V., Noncommutative q-photonadded coherent states, Phys. Rev. A 93053824 (2016). https://doi.org/10.1103/PhysRevA.93.053824

[26] Blitvić, N., The (q,t)-Gaussian process, J. Func. Anal., 263 3270-3305 (2012). https://doi.org/10.1016/j.jfa.2012.08.006. 
[27] Wada, T. and Suyari, H., A two-parameter generalization of ShannonKhinchin axioms and the uniqueness theorem, Phys. Lett. A, $\mathbf{3 6 8} \quad 199-205 \quad$ (2007). https://doi.org/10.1016/j.physleta.2007.04.009.

[28] Gell-Mann, M. and Tsallis, C. (Eds.), Nonextensive Entropy - Interdisciplinary Applications, (Oxford Univ. Press, 2004). https://global.oup.com/academic/product/nonextensive-entropy-9780195159776

[29] Tsallis, C., Introduction to Nonextensive Statistical Mechanics: Approaching a Complex World, (Springer, 2009). http://dx.doi.org/10.1007/978-0-387-85359-8

[30] Kowalski, A. M., Rossignoli, R. D., and Curado, E. M. F. (Eds.), Concepts and Recent Advances in Generalized Information Measures and Statistics, (Bentham Science Pub., 2013). http://dx.doi.org/10.2174/97816080576031130101.

[31] Tsallis, C., Beyond BoltzmannGibbsShannon in physics and elsewhere, Entropy, 21 696-724 (2019). https://doi.org/10.3390/e21070696.

[32] Naudts, J., Generalised Thermostatistics, (Springer, 2011). http://dx.doi.org/10.1007/978-0-85729-355-8

[33] Nonextensive Statistical Mechanics and Thermodynamics. http://tsallis.cat.cbpf.br/biblio.htm http://tsallis.cat.cbpf.br/biblio.htm

[34] Carlitz, L., A degenerate Staudt-Clausen theorem, Arch. Math., 72833 (1956). https://doi.org/10.1007/BF01900520

[35] Carlitz, L., Degenerate Stirling, Bernoulli and Eulerian numbers, Utilitas Math., 15 51-88 (1979).

[36] Balamurugan, M., Chakrabarti, R., and Jagannathan, R., An extension of Bernoulli polynomials inspired by the Tsallis statistics, arXiv:1612.0749 [math-ph]. https://arxiv.org/abs/1612.07496

[37] Gelfand, I. M., Graev, M. I., and Retakh, V. S., General gamma functions, exponentials, and hypergeometric functions, Russian Math. Surveys 53 1-55 (1998). https://doi.org/10.1070/RM1998v053n01ABEH000008. 
[38] Exton, H., q-Hypergeometric Functions and Applications, (Ellis Harwood, 1983). http://cds.cern.ch/record/99100.

[39] Kac, V. and Cheung, P., Quantum Calculus, (Springer, 2002). http://dx.doi.org/10.1007/978-1-4613-0071-7.

[40] Chakrabarti, R. and Jagannathan, R., A $(p, q)$-oscillator realization of two-parameter quantum algebras, J. Phys. A: Math. Gen. 24 L711-L718 (1991). https://doi.org/10.1088/0305-4470/24/13/002.

[41] Jannussis, A., Brodimas, G., and Mignani, I., Quantum groups and Lieadmissible time evolution, J. Phys. A: Math. Gen. 24 (1991) L775-L778. https://doi.org/10.1088/0305-4470/24/14/004.

[42] Arik, M., Demircan, E., Turgut, T., Ekinci, L., and Mungan, M., Fibonacci oscillators, Z. Phys. C: Particles and Fields 55 89-95 (1992). https://doi.org/10.1007/BF01558292.

[43] Jagannathan, R. and Srinivasa Rao, K., Two-parameter quantum algebras, twin-basic numbers, and associated generalized hypergeometric series, arXiv:math/0602613 math.NT]. https://arxiv.org/abs/math/0602613.

[44] Parthasarathy, R. and Viswanathan, K. S., A q-analogue of the supersymmetric oscillator and its $q$-superalgebra, J. Phys. A: Math. Gen. 24 613-618 (1991). https://doi.org/10.1088/0305-4470/24/3/019.

[45] Chaichian, M. and Kulish, P. P., Quantum lie superalgebras and q-oscillators, Phys. Lett. B $234 \quad 72-80 \quad$ (1990). https://doi.org/10.1016/0370-2693(90)92004-3.

[46] Odaka, K., Kishi, T., and Kamefuchi, S., On quantization of simple harmonic oscillators, J. Phys. A: Math. Gen. 24 L591-596 (1991). https://doi.org/10.1088/0305-4470/24/11/004

[47] Chaturvedi, S., Jagannathan, R. and Srinivasan, V., Tamm-Dancoff deformation of bosonic oscillator algebras, Mod. Phys. Lett. A 8 3727-3734 (1993). https://doi.org/10.1142/S0217732393003457.

[48] Gavrilik, A. M. and Rebesh, A. P., A q-oscillator with 'accidental' degeneracy of energy levels, Mod. Phys. Lett. A 22 949-960 (2007). https://doi.org/10.1142/S0217732307022827. 
[49] Gavrilik, A. M. and Rebesh, A. P., Occurrence of pairwise energy level degeneracies in q,p-oscillator model, Ukr. J. Phys. 53 586-594 (2008). http://archive.ujp.bitp.kiev.ua/files/journals/53/6/530611p.pdf.

[50] Gavrilik, A. M. and Rebesh, A. P., Plethora of $q$-oscillators possessing pairwise energy level degeneracy, Mod. Phys. Lett. A, 23 921-932 (2008). https://doi.org/10.1142/S021773230802687X.

[51] Katriel, J. and Quesne, C., Recursively minimallydeformed oscillators, J. Phys. A: Math. Gen. 37 1650-1661 (1996). https://doi.org/10.1063/1.531475.

[52] Burban, I. M., On $(p, q ; \alpha, \beta, l)$-deformed oscillator and its generalized quantum Heisenberg-Weyl algebra, Phys. Lett. A, 366 308-314 (2007). https://doi.org/10.1016/j.physleta.2007.02.051.

[53] Hounkonnou, M. N. and Ngompe Nkouankam, E. B., On $\left(p, q, \mu, \nu, \phi_{1}, \phi_{2}\right)$-generalized oscillator algebra and related bibasic hypergeometric functions, J. Phys. A: Math. Theor. 40 8835-8844 (2007). https://doi.org/10.1088/1751-8113/40/30/015.

[54] Gavrilik, A. M., Kachurik, I. I., and Rebesh, A. P., QuasiFibonacci oscillators, J. Phys. A: Math. Theor. 43245204 (2010). https://doi.org/10.1088/1751-8113/43/24/245204.

[55] Chung, W. S. and Hassanabadi, H., $\varphi$-deformed boson algebra based on $\varphi$-deformed addition and non-classical properties of $\varphi$-deformed coherent states, Phys. Scr. 95035106 (2020). https://doi.org/10.1088/1402-4896/ab4f94

[56] Mursaleen, M., Khan, F., and Khan, A., Approximation by $(p, q)$-Lorentz polynomials on a compact disk, Complex Anal. Oper. Theory, $10 \quad 1725-1740 \quad$ (2016). https://doi.org/10.1007/s11785-016-0553-4.

[57] Alotaibi, A., Nasiruzzaman, M., and Mursaleen, M., A Dunkl type generalization of Szász operators via postquantum calculus, J. Inequal. Appl. 2018:287 (2018). https://doi.org/10.1186/s13660-018-1878-5 
[58] Soontharanon, J. and Sitthiwirattham, T., On fractional $(p, q)$-calculus, Adv. Difference Equations, 2020:35 (2020). https://doi.org/10.1186/s13662-020-2512-7.

[59] Manko, V. I., Marmo, G., Sudarshan, E. C. G., and Zaccaria, F., foscillators and nonlinear coherent states, Phys. Scr. 55 528-541 (1997). https://doi.org/10.1088/0031-8949/55/5/004.

[60] Dudinets, I. V., Man'ko, V. I., Marmo, G., and F Zaccaria, F., Tomography on f-oscillators, Phys. Scr. 92115101 (2017). https://doi.org/10.1088/1402-4896/aa8e22

[61] Man'ko, V. I., Marmo, G., Solimeno, S., and Zaccaria, F., Physical nonlinear aspects of classical and quantum q-oscillators, Int. J. Mod. Phys. A $\mathbf{8}, \quad 3577-3597$ (1993). https://doi.org/10.1142/S0217751X93001454

[62] Man'ko, V. I., Marmo, G., Solimeno, S., and Zaccaria, F., Correlation functions of quantum q-oscillators., Phys. Lett. A 176 173-175 (1993). https://doi.org/10.1016/0375-9601(93)91029-5

[63] Man'ko, V. I. and Tino, G. M., Experimental limit on the blue shift of the frequency of light implied by a $q$-nonlinearity, Phys. Lett. A 202 24-27 (1995). https://doi.org/10.1016/0375-9601(95)00327-Y

[64] Lerner, E. C., Huang, H. W., and Walters, G. E., Some mathematical properties of oscillator phase operators, J. Math, Phys., 11 1679-1684 (1970). https://doi.org/10.1063/1.1665310.

[65] Sudarshan, E.C.G., Diagonal harmonious state representations, Internat. J. Theor. Phys., $32 \quad$ 1069-1076 (1993). https://doi.org/10.1007/BF00671789.

[66] Dodonov, V. V. and Mizrahi, S. S., Uniform nonlinear evolution equations for pure and mixed quantum States, Ann. Phys. 237 226-268 (1995). https://doi.org/10.1006/aphy.1995.1010.

[67] Dodonov, V. V., Nonclassical states in quantum optics: A squeezed review of the first 75 years, J. Opt. B: Quantum Semiclass. Opt., 4 R1 (2002). https://doi.org/10.1088/1464-4266/4/1/201. 
[68] Borges, E. P., On a q-generalization of circular and hyperbolic functions, J. Phys. A: Math. Gen. 31 5281-5288 (1998). https://doi.org/10.1088/0305-4470/31/23/011.

[69] Bendjeffal, A., Smida, A., Messamahb, J., and Hachemanec, M., A class of nonlinear coherent states attached to Tsallis q-exponential, Eur. Phys. J. Plus 134:330 (2019). https://doi.org/10.1140/epjp/i2019-12865-9.

[70] Jaganathan, R. and Sinha, S., A $q$-deformed nonlinear map, Phys. Lett. A $338 \quad 277-287 \quad$ (2005). https://doi.org/10.1016/j.physleta.2005.02.042.

[71] Patidar, V. and Sud, K. K., A comparative study on the coexisting attractors in the Gaussian map and its $q$-deformed version, Commun. Nonlin. Sci. Numer. Simul. 14 827-838 (2009). https://doi.org/10.1016/j.cnsns.2007.10.015.

[72] Patidar, V., Purohit, G., and Sud, K. K., Dynamical Behavior of $q$ deformed Henon map, Internat. J. Bifur. Chaos, 21, 1349-1356 (2011). https://doi.org/10.1142/S0218127411029215.

[73] Banerjee, S. and Parthasarathy, R., A q-deformed logistic map and its implications, J. Phys. A: Math. Theor. 44045104 (2011). https://doi.org/10.1088/1751-8113/44/4/045104.

[74] Behnia, S., Yahyavi, M., and Habibpourbisafar, R., Watermarking based on discrete wavelet transform and $q$-deformed chaotic map, Chaos, Solitons \& Fractals $^{6-17} \mathbf{1 0 4}$ (2017). https://doi.org/10.1016/j.chaos.2017.07.020.

[75] Balakrishnan, J., Iyengar, S. V., and Kurths, J., Missing cycles: Effect of climate change on population dynamics, Ind. Acad. Sci. Conf. Series 1 93-99 (2017). https://www.ias.ac.in/article/fulltext/conf/001/01/0093-0099.

[76] Canovas, J. and Munoz-Guillermo, M., On the dynamics of the q-deformed logistic map, Phys. Lett. A 383 1742-1754 (2019). https://doi.org/10.1016/j.physleta.2019.03.003. 
[77] Pourahmadi, M., Taylor Expansion of $\exp \left(\sum_{k=0}^{\infty} a_{k} z^{k}\right)$ and Some Applications Amer. Math. Monthly 91 303-307 (1984). https://doi.org/10.1080/00029890.1984.11971411.

[78] Sachkov, V. N., Combinatorial Methods in Discrete Mathematics, (Cambridge Univ. Press, 1996). https://doi.org/10.1017/CB09780511666186.

[79] Quesne, C., Disentangling $q$-Exponentials: A General Approach, Internat. J. Theor. Phys. 43 545-559 (2004). https://doi.org/10.1023/B:IJTP.0000028885.42890.f5.

[80] Chung, W. S., The new type of extended uncertainty principle and some applications in deformed quantum mechanics, Internat. J. Theor. Phys., $58 \quad 2575-2591$ (2019). https://doi.org/10.1007/s10773-019-04146-z

[81] Andrews, G. E., Askey, R., and Roy, R., Special functions, Encyclopedia of Mathematics and its Applications 71, (Cambridge Univ. Press, 1999). ISBN 978-0-521-78988-2

[82] Chung, W. S., Gavrilik, A. M., and Nazarenko, A. V., Photon gas at the Planck scale within the doubly special relativity, Physica A: Stat. Mech. Appl. 533121928 (2019). https://doi.org/10.1016/j.physa.2019.121928

[83] Jannussis, A., New deformed Heisenberg oscillator, J. Phys. A: Math. Gen. 26 L233-L238 (1993). https://doi.org/10.1088/0305-4470/26/5/011.

[84] Chakrabarti, R., Chandrashekar, R., and Naina Mohammed, S. S., Nonextensive statistics of the classical relativistic ideal gas, Physica A: Stat. Mech. Appl. 389 1571-1584 (2010). https://doi.org/10.1016/j.physa.2009.12.040.

[85] Kim, S., Chung, W. S., and Hassanabadi, H., q-deformed Gamma function, $q$-deformed probability distributions and $q$-deformed statistical physics based on Tsallis $q$-exponential function, Eur. Phys. J. Plus 134 572 (2019). https://doi.org/10.1140/epjp/i2019-13082-4. 
[86] Borges, E. P., A possible deformed algebra and calculus inspired in nonextensive thermostatistics, Physica A: Stat. Mech. Appl. 340 95-101 (2004). https://doi.org/10.1016/j.physa.2004.03.082.

[87] Chung, W. S., Two different interpretations of $q$-boson algebra and a new q-deformed statistical physics, Phys. Scr., 94115001 (2019). https://doi.org/10.1088/1402-4896/ab2caa.

[88] Rebesh, A. P., Gavrilik, A. M., and Kachurik, I. I., Elements of $\mu-$ Calculus and Thermodynamics of $\mu$-Bose Gas Model, Ukr. J. Phys. 58 1182-1191 (2013). https://doi.org/10.15407/ujpe58.12.1182.

[89] Schwämmlea, V. and Tsallis, C., Two-parameter generalization of the logarithm and exponential functions and BoltzmannGibbs-Shannon entropy, J. Math. Phys., 48113301 (2007). https://doi.org/10.1063/1.2801996 